\title{
DECLARACIONES DE CONFLICTOS DE INTERÉS EN LAS PUBLICACIONES BIOMÉDICAS. ESTUDIO DE CUATRO REVISTAS CLÍNICAS ESPAÑOLAS
}

\begin{abstract}
Francesc Roig ${ }^{1}$, Ángel Borrego ${ }^{2}$
Resumen: La declaración de las relaciones financieras y personales de los investigadores con las compañías farmacéuticas es percibida como un paso esencial en la gestión de los potenciales conflictos de interés. El objetivo del estudio fue evaluar las declaraciones de conflictos de interés en cuatro revistas biomédicas españolas publicadas en castellano: Atención Primaria, Medicina Clínica, Revista Clínica Española y Revista Española de Cardiología. Se analizaron las publicaciones de 2012 calificadas por las revistas como "Editorial”, "Artículo original”, "Documento de consenso", "Revisión" y "Artículo especial”. De 412 artículos, 315 (76\%) presentaron declaración de conflictos de interés. Por revista, el 73\% de los artículos de Atención Primaria, el 68\% de Medicina Clínica, el 60\% de Revista Clínica Española y el 100\% de Revista Española de Cardiología tenía declaración de conflictos de interés. Solo el 12\% de las declaraciones describía alguna actividad susceptible de generar conflicto de interés, mientras que el resto afirmaba no tener. Además de una variabilidad considerable en la frecuencia de declaración de conflictos de interés, la presencia de actividades declaradas es baja. Parece necesario mejorar la uniformidad y transparencia de las políticas de declaración de conflictos de interés.
\end{abstract}

Palabras clave: conflictos de interés, revistas biomédicas, política editorial, Atención Primaria

\section{Conflict of interest statements in biomedical publications. A study of four Spanish health care journals}

\begin{abstract}
Disclosure of financial relationships between researchers and pharmaceutical companies is seen as an essential step in the management of potential conflicts of interest. The objective of the study was to evaluate the frequency of conflicts of interest disclosure in four Spanish biomedical journals: Atención Primaria, Medicina Clínica, Revista Clínica Española and Revista Española de Cardiología. Publications qualified as "Editorial", "Original Article", "Consensus Document", "Review" and "Special article" in 2012 were analyzed. Of 412 articles, 315 (76\%) had a conflict of interest statement. By journal, 73\% of the articles in Atención Primaria, 68\% in Medicina Clínica, 60\% in Revista Clinica Española and 100\% in Revista Española de Cardiología had disclosure of conflicts of interest. Only $12 \%$ of disclosures reported activities, while the rest maintains "No conflict of interest". In addition to a considerable variability in the frequency of conflicts of interest disclosure, the presence of activities disclosed is low. It would be necessary to improve the consistency and transparency of the policy declaration of conflicts of interest.
\end{abstract}

Key words: Conflicts of interest, Biomedical journals, Editorial Policy, Primary Care

Declaraçóes de conflitos de interesse em publicaçóes biomédicas. Estudo de quatro revistas clínicas espanholas

Resumo: A declaração das relações financeiras e pessoais de pesquisadores com empresas farmacêuticas é vista como um passo essencial na gestáo dos potenciais conflitos de interesse. O objetivo do estudo foi avaliar as declaraçóes de conflitos de interesses em quatro revistas biomédicas espanholas publicadas em castelhano: Atención Primaria, Medicina Clínica, Revista Clínica Española e Revista Española de Cardiología. Foram analisadas as publicaçôes de 2012 classidicadas pelas revistas enquanto: "Editor", "Artigo Original", "Documento de consenso", "Revisão" e "Artigo especial". De 412 artigos, 315 (76\%) apresentaram declaração de conflitos de interesses. Por revista, 73\% dos artigos de Atención Primaria, 68\% de Medicina Clínica, 60\% da Revista clínica Española e 100\% da Revista Espanhola de Cardiologia tinham declaração de conflitos de interesses. Apenas 12\% das declraçóes descrevia qualquer atividade suscetível a gerar conflito de interesses, enquanto o resto alegou não ter. Ademais de uma considerável variabilidade na frequência de declaração de conflitos de interesses, a presença de atividades declaradas é baixa. Parece necessário melhorar a uniformidade e a transparência das políticas de declaração de conflito de interesses.

Palavras-chave: conflitos de interesse, revistas biomédicas, política editorial, atenção primária à saúde

\footnotetext{
${ }^{1}$ Medical Information \& Communication, Novartis Farmacéutica, Barcelona, Espańa Correspondencia: frolos62@gmail.com

${ }^{2}$ Facultat de Biblioteconomia i Documentació, Universitat de Barcelona, Barcelona, España
} 


\section{Introducción}

\section{The actual product of the pharmaceutical industry is knowledge, pills and prescription ointment are no more than packaging for knowledge. (Drucker, 1993, p 166)}

En su obra Post-capitalist society(1) Peter Drucker afirma que el producto final de la industria farmacéutica es el conocimiento, y que cápsulas y comprimidos son solo la forma como este conocimiento se empaqueta. Con esta afirmación no está solamente creando una abstracción teórica con la que pretende describir las características diferenciales de lo que él llama "capitalismo informacional"; está señalando también un hecho que tiene profundas implicaciones en el ámbito de la salud pública. Lo que significa la afirmación de Drucker es que cuando alguien va a la farmacia y adquiere una caja de aspirinas no está pagando por un conjunto de unidades de $500 \mathrm{mg}$ de ácido acetilsalicílico comprimido, envasadas en un blíster de aluminio. Lo que busca, aquello por lo que está dispuesto a asumir su coste económico, es el conocimiento de que cada una de esas unidades, tomada de determinada manera y en determinadas circunstancias, tendrá determinados efectos. Es la combinación del conocimiento bioquímico acumulado a lo largo del desarrollo de un fármaco, empaquetado en la forma farmacéutica que se comercializa, junto al conocimiento de la enfermedad que tienen los profesionales de la salud, lo que permite avanzar en el cuidado de la salud y en la obtención de tratamientos cada vez más efectivos(2). Es, pues, en el conocimiento donde radica el valor del fármaco, y es en la producción de este conocimiento donde la industria invierte gran cantidad de los recursos que utiliza. Pero la generación, empaquetamiento y comercialización de conocimiento científico en forma de medicamentos no resulta suficiente para conseguir ni la extensión de los esperados beneficios sociales que debe provocar su uso, ni el buscado retorno de la inversión. Para que este conocimiento científico consiga generar valor para la companía que lo ha creado, para la comunidad médica que debe utilizarlo y para la sociedad en su conjunto, es necesario comunicarlo extensivamente, asegurar que los médicos que pueden utilizar el fármaco en el tratamiento de sus pacientes conozcan sus propiedades y los beneficios que aporta al arsenal terapéutico ya existente.

Dado que este conocimiento va destinado a la comunidad médica, su comunicación debe seguir un proceso específico y altamente estandarizado, que se basa en la publicación en revistas especializadas siguiendo el proceso de evaluación de expertos (peer review). De forma resumida, en este modelo, todo trabajo de investigación que se presenta para ser publicado en una revista científica debe pasar primero la revisión y evaluación de expertos en el ámbito de estudio, con el objetivo de validar tanto su justificación y necesidad como la metodología empleada, los resultados y la aportación nueva al conocimiento. A petición del editor de la revista en la que se ha presentado el original con el objetivo de ser publicado, los expertos revisores hacen los comentarios y sugerencias de mejora que creen convenientes y aconsejan sobre la conveniencia o no de publicarlo. En un proceso donde el editor conserva la última palabra, el manuscrito original puede ser aceptado directamente, aceptado condicionalmente si se introducen determinados cambios, rechazado, pero indicando cambios que permitirían una nueva evaluación, o rechazado sin opción a nueva revisión. Por medio de este particular sistema, autorregulado por la propia comunidad científica, el conocimiento generado en el desarrollo y uso de un fármaco se difunde entre la comunidad médica a la que se dirige vía las revistas especializadas.

De este modo, en la investigación clínica destinada a hacer avanzar el conocimiento sobre el abordaje y el tratamiento de las enfermedades se crea un escenario con tres actores clave: los médicos, que pueden ser investigadores y productores de conocimiento a la vez que destinatarios directos del nuevo conocimiento generado; la industria farmacéutica, financiadora de la producción de un conocimiento que comercializa después en forma de medicamento, y las revistas científicas, canal de comunicación y difusión del conocimiento producido. Los tres actores establecen vínculos de cooperación necesaria en beneficio mutuo que, a lo largo del tiempo, han ido ganando en interrelación y complejidad. Para los médicos la cooperación supone, por una parte, oportunidades para hacer investigación, de promoción y 
reconocimiento profesional, de participación en congresos y visibilidad pública, además de beneficios económicos por actividades de consultoría, asesoramiento y formación; por otra, les ofrece acceso a las novedades médicas. Para la industria supone la posibilidad de aumentar la visibilidad y el conocimiento de sus productos, posicionándose como referentes ante la comunidad médica prescriptora y por tanto posibilitando un mayor uso que repercutirá en aumento de las ganancias económicas. Para las revistas científicas, la publicación de estudios que incluyen gran número de pacientes, altamente leídos y referenciados, y que pueden hacer aumentar su factor de impacto, además de conllevar la comercialización de otros productos, como suplementos o monografías financiadas directamente por la industria, copias de artículos para distribuir entre los profesionales en actividades promocionales, o la propia contratación de espacios publicitarios, que generarán considerables ganancias económicas.

Pero a pesar de los efectos beneficiosos de esta colaboración, es necesario tener presente que los objetivos de la industria y de los profesionales de la salud son diferentes. El profesional de la salud tiene como interés principal actuar por el bien de las personas y la comunidad a la que sirve(3). Las compañías farmacéuticas, en cambio, generar beneficios que permitan la retribución de los empleados, la recuperación de las inversiones y mantener la viabilidad futura de la compañía, y hacerlo aportando novedades terapéuticas en la sociedad. A esta situación se añaden las necesidades e intereses del sector de la edición científica, para lo que la publicación de originales de estudios financiados por la industria farmacéutica ofrece considerables oportunidades de negocio que a menudo son fundamentales para asegurar su rentabilidad. Como consecuencia, cuando los profesionales de la salud colaboran con la industria, investigando y publicando en revistas científicas, pueden encontrarse ante situaciones de conflicto de intereses, en las que aparecen intereses secundarios que los alejan del objetivo fundamental en beneficio de la sociedad.

Es conocido que los artículos peer-reviewed son el principal medio por el que los resultados de la investigación médica influyen en la práctica clínica(4). Pero además de influenciar la práctica clínica, la investigación publicada en las revistas científicas también actúa definiendo el ámbito de las inquietudes médicas, estableciendo lo que se considera como "conocimiento científico" dentro de una disciplina(5) y constituyendo pues un elemento fundamental en los procesos de toma de decisiones, tanto médicas como políticas y de salud pública. En este contexto, la existencia de intereses propios, diferentes al bienestar del paciente y la integridad de la investigación como intereses principales de la profesión médica, intereses que pueden entrar en conflicto con la atención al paciente y la salud de la sociedad, conlleva el riesgo de minar la confianza en la evidencia publicada $(6,7)$, tanto por los profesionales de la salud como por los decisores y ciudadanos. Por ello, la declaración de las relaciones financieras y personales de los investigadores con las compañías farmacéuticas es percibida como un paso esencial en la gestión de los potenciales conflictos de interés (8) y las revistas biomédicas están adoptando desde hace cierto tiempo la solicitud de declaración de posibles conflictos de interés como requerimiento imprescindible para los autores que someten su manuscrito al proceso de revisión para su publicación. Recientemente se han analizado las políticas de gestión de conflictos de intereses en las revistas biomédicas clínicas españolas publicadas en castellano incluidas en Journal Citation Report (JCR), observando que la mayoría de revistas incluyen en sus instrucciones para autores al menos una mención a la necesidad de declarar los conflictos de interés y ofrecen algún tipo de descripción de estas situaciones, si bien se trata de definiciones laxas sobre las relaciones económicas que se deben declarar, y sin que especifiquen el alcance de las relaciones personales(9). El estudio que se presenta tiene como objetivo analizar la implementación efectiva de estas políticas en cuatro revistas seleccionadas.

\section{Metodología}

Dado que la publicación de nuevo conocimiento científico tiene como objetivo fundamental influenciar en la práctica médica, y ya que el mayor grupo de prescriptores se encuentra entre los médicos de familia, se seleccionaron las revistas españolas publicadas en castellano en la sección "Medicine, General \& Internal" del JCR 2011, por su contenido eminentemente clínico y su 
orientación generalista: Atención Primaria, Medicina Clínica y Revista Clínica Española. La idoneidad de la elección de las revistas estaba confirmada por los resultados del estudio de González de Dios y colaboradores(10), indicando que de las 22 revistas médicas en castellano que los médicos de familia consultan con regularidad Atención Primaria y Medicina Clínica son las únicas indexadas en las bases de datos bibliográficas más importantes (PubMed y ISI-Web of Knowledge). Por otra parte, para completar la visión sobre la comunicación de los conflictos de interés se consideró importante incluir alguna revista especializada. Dado que las enfermedades cardiovasculares son las más prevalentes y las que causan mayor mortalidad(11), y que son enfermedades muy frecuentemente tratadas por los médicos de atención primaria, se seleccionó la única revista española en castellano incluida en JCR en la sección "Cardiac \& Cardiovascular Systems", manteniendo el criterio de máxima calidad científica: Revista Española de Cardiología.

Así pues, el análisis sobre las declaraciones de conflictos de interés se realizó sobre cuatro revistas: Atención Primaria, Medicina Clínica, Revista Clínica Española y Revista Española de Cardiología. Entre febrero y marzo de 2013 se descargaron de los sitios web los números ordinarios publicados durante 2012, excluyendo suplementos, números especiales y monografías. Se analizaron las publicaciones que las revistas calificaban como "Editorial", "Artículo original", "Documento de consenso", "Revisión" y "Artículo especial". De cada publicación se extrajeron los siguientes datos: tipo, autores, inclusión o no de un apartado con la declaración de conflicto de intereses, declaración o no de algún conflicto de interés, y conflictos de interés declarados. Se analizaron los datos con estadísticas de resumen que describen la frecuencia de declaración de conflicto de interés (DCI) según revista y tipo de publicación. Finalmente, se comparó la presencia o ausencia de DCI por revista y por tipo de publicación y la presencia o ausencia de conflicto de interés en aquellos artículos que presentaban DCI utilizando la prueba de la Chi-cuadrado, considerando un valor de $\mathrm{p}$ menor de 0,05 como estadísticamente significativo. Las cuatro revistas tienen como requerimiento en las "Instrucciones para autores" la necesidad de realizar DCI al enviar un manuscrito a revisión.

\section{Resultados}

Se analizó un total de 412 publicaciones aparecidas como editoriales, artículos originales, revisiones, documentos de consenso o artículos especiales a lo largo de 2012, distribuidas de la siguiente forma: 84 (20\%) en Atención Primaria, 184 (45\%) en Medicina Clínica, 40 (10\%) en Revista Clínica Española y 104 (25\%) en Revista Española de Cardiología. La tabla 1 recoge el número de artículos según tipología y revista.

Del total de los artículos analizados, 315 (76\%) incluían DCI. Por revista, en Atención Primaria 61 artículos (73\%) incluyen DCI, en Medicina Clínica 126 (68\%), en Revista Clínica Española $24(60 \%)$ y la totalidad de los 104 artículos publicados en Revista Española de Cardiología (100\%) presentaban DCI, diferencias que solo alcanzaron la significación estadística entre Revista Española de Cardiología y el resto ( $\mathrm{p}<0,0001$ con cada una).

Tabla 1: Tipología de artículos por revista

\begin{tabular}{|l|l|l|l|l|l|}
\hline & Atención Primaria & Medicina Clinica & $\begin{array}{l}\text { Revista Clinica } \\
\text { Española }\end{array}$ & $\begin{array}{l}\text { Revista Española de } \\
\text { Cardiología }\end{array}$ & Total (\%) \\
\hline $\begin{array}{l}\text { A r t í c u l o s } \\
\text { originales }\end{array}$ & 56 & 91 & 27 & 66 & $240(58 \%)$ \\
\hline Editoriales & 23 & 48 & 12 & 35 & $118(29 \%)$ \\
\hline Revisiones & -- & 35 & -- & 3 & $38(9 \%)$ \\
\hline $\begin{array}{l}\text { D o cu me n to s } \\
\text { consenso }\end{array}$ & 3 & 5 & 1 & -- & $9(2 \%)$ \\
\hline $\begin{array}{l}\text { A r t í c u l o s } \\
\text { especiales }\end{array}$ & 2 & 5 & -- & -- & $7(2 \%)$ \\
\hline Total & 84 & 184 & 40 & 104 & 412 \\
\hline
\end{tabular}


Tabla 2: Artículos con DCI por revista y tipo de articulo. DCI: Declaración de conflictos de interés

\begin{tabular}{|l|l|l|l|l|l|}
\hline & $\begin{array}{l}\text { A tención } \\
\text { Primaria }\end{array}$ & Medicina Clinica & $\begin{array}{l}\text { Revista Clinica } \\
\text { Española }\end{array}$ & $\begin{array}{l}\text { Revista Española de } \\
\text { Cardiología }\end{array}$ & Total \\
\hline $\begin{array}{l}\text { Artículos originales } \\
\text { con DCI }\end{array}$ & $53(95 \%)$ & $85(93 \%)$ & $23(85 \%)$ & $66(100 \%)$ & $227(96 \%)$ \\
\hline Editoriales con DCI & $4(17 \%)$ & $0(0 \%)$ & $0(0 \%)$ & $35(100 \%)$ & $39(33 \%)$ \\
\hline Revisiones con DCI & -- & $33(94 \%)$ & -- & $3(100 \%)$ & $36(95 \%)$ \\
\hline $\begin{array}{l}\text { D o c u m e n t o s } \\
\text { consenso con DCI }\end{array}$ & $3(100 \%)$ & $3(60 \%)$ & $1(100 \%)$ & -- & $7(78 \%)$ \\
\hline $\begin{array}{l}\text { Artículos especiales } \\
\text { con DCI }\end{array}$ & $1(50 \%)$ & $5(100 \%)$ & -- & -- & $6(86 \%)$ \\
\hline Total DCI & $61(73 \%)$ & $126(68 \%)$ & $24(60 \%)$ & $104(100 \%)$ & $315(76 \%)$ \\
\hline
\end{tabular}

En función de la tipología, 227 (96\%) artículos originales, $39(33 \%)$ editoriales, $36(95 \%)$ revisiones, 7 (78\%) documentos de consenso y 6 $(86 \%)$ artículos especiales incluían DCI. Al comparar los dos únicos tipos que se encuentran en las cuatro revistas, artículos originales y editoriales, la diferencia en la presencia de DCI fue estadísticamente significativa $(p<0,0001)$. En la tabla 2 se detalla la presencia de DCI según tipo de publicación para cada revista.

De los 315 artículos que presentaban DCI, 278 (88\%) afirmaban explícitamente que los autores no tenían ninguno, mientras que 37 (12\%) declaraban actividades susceptibles de generar conflicto de interés. Por revista, declararon no existencia de conflicto de interés 58 artículos (95\%) en Atención Primaria, 111 (87\%) en Medicina Clínica, 22 (83\%) en Revista Clínica Española y 87 (84\%) en Revista Española de Cardiología, sin que las diferencias entre revistas fueran estadísticamente significativas $(\mathrm{p}=0,177)$.

Por tipo de artículo, los autores del 85\% de los artículos originales declaraban no tener ningún conflicto de interés, así como los del 75\% de los

Tabla 3: Artículos con DCI y con CI reportada por tipo de artículo y revista. DCI: Declaración de conflictos de interès. CI: Conflicto de interés

\begin{tabular}{|l|l|l|l|l|}
\hline & $\begin{array}{l}\text { Atención } \\
\text { Primaria }\end{array}$ & Medicina Clinica & $\begin{array}{l}\text { Revista Clínica } \\
\text { Española }\end{array}$ & $\begin{array}{l}\text { Revista Española de } \\
\text { Cardiologia }\end{array}$ \\
\hline Editoriales & 23 & 48 & 12 & 35 \\
\hline Con DCI & $4(17 \%)$ & $0(0 \%)$ & $0(0 \%)$ & $35(100 \%)$ \\
\hline Reportando CI & $0(0 \%)$ & -- & -- & $10(29 \%)$ \\
\hline Artículos originales & 56 & 91 & 27 & 66 \\
\hline Con DCI & $53(95 \%)$ & $85(93 \%)$ & $23(85 \%)$ & $66(100 \%)$ \\
\hline Reportando CI & $1(2 \%)$ & $12(14 \%)$ & $2(9 \%)$ & $7(11 \%)$ \\
\hline Revisiones & -- & 35 & - & 3 \\
\hline Con DCI & -- & $33(94 \%)$ & -- & $3(100 \%)$ \\
\hline Reportando CI & -- & $1(3 \%)$ & -- & $0(0 \%)$ \\
\hline $\begin{array}{l}\text { Documentos } \\
\text { Consenso }\end{array}$ & 3 & 5 & 1 & -- \\
\hline Con DCI & $3(100 \%)$ & $3(60 \%)$ & $1(100 \%)$ & -- \\
\hline Reportando CI & $2(67 \%)$ & $1(33 \%)$ & $0(0 \%)$ & -- \\
\hline Artículos Especiales & 2 & 5 & -- & -- \\
\hline Con DCI & $1(50 \%)$ & $5(100 \%)$ & -- & -- \\
\hline Reportando CI & $0(0 \%)$ & $1(20 \%)$ & -- & -- \\
\hline
\end{tabular}


editoriales, el $92 \%$ de las revisiones, el $67 \%$ de los documentos de consenso y el $28 \%$ de los artículos especiales. Las diferencias entre los dos tipos de publicaciones presentes en las cuatro revistas, artículos originales y editoriales, fueron estadísticamente significativas $(\mathrm{p}=0,0047)$. La tabla 3 detalla la presencia o no de conflictos de interés según el tipo de artículo y revista.

\section{Discusión}

Hasta donde conocemos, este es el primer estudio que tiene como objetivo determinar la frecuencia de las declaraciones de conflicto de interés en revistas españolas. El 76\% de frecuencia de DCI en las revistas analizadas se encuentra en la franja alta de la revelada en estudios realizados en otros países. Así, al examinar las revistas especializadas en gastroenterología presentes en el JCR en 2005 se observó que tenían DCI el $42 \%$ en los artículos originales(12). Esta frecuencia disminuía a un $25 \%$ en los estudios sobre estents coronarios publicados en revistas afiliadas al International Committee of Medical Journal Editors(13) (ICMJE) y se situaba en el $44 \%$ en los artículos de investigación en el ámbito del VIH publicados en revistas asociadas al ICMJE(14). En los artículos originales publicados en 2008 y 2009 en seis revistas de cirugía vascular(15) se observó una frecuencia media de DCI del 20\%. En la literatura de cuidados paliativos en oncología publicada entre 2004 y 2009 se observó que el $51 \%$ tenía $\mathrm{DCI}(16)$. Más recientemente, al estudiar las políticas de DCI en las revistas biomédicas en Corea se observó que el 57\% de los artículos contenían DCI, aunque el análisis se limitó a una muestra de 144 correspondientes a ensayos clínicos publicados en inglés (17). Finalmente, la frecuencia de publicación de DCI en los ensayos clínicos aleatorizados y controlados publicados en las principales revistas de cirugía entre 2005 y 2010 fue del 25\%(18).

Al interpretar los resultados del presente estudio hay que tener en cuenta que la media del $76 \%$ de DCI aumenta hasta el $96 \%$ cuando se limita a artículos originales. Al centrar el análisis en las revisiones, se observa que solo dos de las revistas analizadas han publicado este tipo de artículos durante 2012, un total de 38, el $95 \%$ del cual presentaba DCI, una frecuencia similar a la de los artículos originales (96\%; sin diferen- cia significativa, $\mathrm{p}<0,969)$. Esta cifra es superior al 81,7\% encontrado(19) en un reciente análisis de las DCI en JAMA y NEJM, situando también la frecuencia de las DCI en las revisiones de las revistas españolas analizadas en la banda alta de la identificada en la literatura internacional. En cuanto a los editoriales, en cambio, solo el 33\% presenta DCI, una frecuencia significativamente menor que la observada en los artículos originales y revisiones $(96 \%, p<0,0001 ; 95 \%, p<0,0001$, respectivamente). Esta cifra se encuentra como mínimo 10 puntos por debajo de lo conocido en la literatura: el $51 \%$ en un estudio que evaluó la frecuencia de DCI en editoriales sobre ensayos clínicos en el Journal of Clinical Oncology(20), el $46 \%$ detectado en las revistas especializadas en gastroenterología presentes en el JCR en 20056, el $43 \%$ observado en los editoriales publicados en las cuatro revistas oncológicas más importantes(21) y el más reciente $47 \%$ hallado en los editoriales relacionados con estudios clínicos de Fase III en seis revistas oncológicas(22).

Además de la frecuencia de las DCI, la insistencia con que estas contienen situaciones concretas susceptibles de generar conflictos de interés, así como la tipología de estas situaciones, son también relevantes para obtener una comprensión adecuada de su significado real. Recientemente, miembros de la junta directiva de Osatzen(23) mostraban su sorpresa por la afirmación de ausencia de conflictos de interés de los autores de un documento de posicionamiento de sociedades científicas de atención primaria sobre las barreras de prescripción de los anticoagulantes y de un suplemento monográfico que incluía el tratamiento de la artrosis, hecho que contraponían a las numerosas publicaciones y colaboraciones entre los autores de ambos artículos con las empresas farmacéuticas que comercializaban fármacos en esas áreas. Aunque de forma general la frecuencia con que la DCI está presente en las revistas analizadas es relativamente elevada, son muy pocas las que realmente declaran actividades susceptibles de generar conflicto de interés. Solo el 12\% de las publicaciones que tienen DCI declaran alguna actividad. En la literatura se encuentran grandes diferencias en cuanto a la frecuencia con que las DCI describen relaciones susceptibles de generar conflictos de interés. Así, si bien la frecuencia en 
las cuatro revistas españolas es casi el doble del $6,5 \%$ observado en las revistas especializadas en gastroenterología en 2006, se encuentra en cambio muy por debajo del $88 \%$ identificado recientemente en los ensayos clínicos aleatorizados publicados en las principales revistas de cirugía entre 2005 y 2010.

Lógicamente, una parte relevante de las diferencias viene provocada por el tipo de publicación. La realización de un ensayo clínico aleatorizado requiere unos recursos económicos a menudo considerables y los autores de las publicaciones suelen ser los coordinadores del estudio, profesionales considerados líderes de opinión en el sector, con lazos frecuentes y conocidos con la industria. Es esperable, pues, que este tipo de publicaciones sean más transparentes en cuanto a las DCI de los autores. En la muestra estudiada, solo 6 publicaciones eran ensayos clínicos aleatorizados, sin que ninguna de ellas correspondiera a una intervención farmacológica. Y aunque las 6 presentaban DCI, en todas ellas los autores manifestaban que no tenían ninguno.

El estudio realizado presenta varias limitaciones. $\mathrm{Al}$ analizarse 4 revistas, y vista la elevada variabilidad entre ellas, la frecuencia de DCI y las tipologías observadas podría no reflejar la situación general de las revistas españolas. Por otra parte, la elección de la tipología de artículos condiciona los resultados ya que, como se ha observado, varían dentro de la muestra en función del tipo de publicación. Sin embargo, el trabajo ha analizado las dos tipologías presentes siempre en todas en las revistas biomédicas, los editoriales y los artícu- los originales, que de acuerdo con la literatura son las más sensibles a reflejar posibles conflictos de interés de sus autores y en las que estos conflictos tienen mayor impacto. Al analizar las tipologías de DCI hay que tener en cuenta que, al no existir definiciones estándar consensuadas de cuáles son y qué significa cada una de ellas, es posible que lo que un autor declara como "Ponencias" otro lo declare como "Presentaciones", aunque en realidad se trate de acciones similares. Sin embargo, la categorización ofrece una muestra altamente ilustrativa del tipo de actividades que actualmente están realizando los médicos en relación con la industria y que pueden ser susceptibles de generar conflictos de interés.

Como conclusión, el estudio revela una considerable variabilidad en la frecuencia de DCI entre las cuatro revistas biomédicas españolas publicadas en castellano analizadas, tanto entre revistas como entre tipología de artículos. También revela una considerable baja frecuencia en la declaración real de actividades susceptibles de generar conflicto de interés por parte de los autores. Parece necesario que las revistas establezcan políticas más uniformes, claras y transparentes de DCI, estableciendo no solo la necesidad de declarar los conflictos, sino el tipo de actividades que son susceptibles de generarlos, porque de lo contrario es poco probable que los propios autores sean capaces de identificarlos y reportarlos. Es responsabilidad de los editores establecer un sistema de alta calidad para las DCI, estandarizando lo que debe ser reportado y asegurando su publicación de forma clara, comprensible y coherente. 
Declaraciones de conflictos de interés en las publicaciones biomédicas - Francesc Roig, Ángel Borrego

\section{Referencias}

1. Drucker P. Post-capitalist society. Oxford: Butterworth-Heineman Ltd., Linacre House, Jordan Hill; 1993.

2. Kreiner K, Tryggesta K. The co-production of chip and society: unpacking packaged knowledge. Scand J Mgmt 2002: 18; 421-449.

3. La Rosa Rodríguez E. Los conflictos de interés. Acta Bioethica 2011: 17(1): 47-54.

4. Krzyzanowska MK, Pintilie M, Tannock IF. Factors associated with failure to publish large randomized trials presented at an oncology meeting. JAMA 2003: 290(4): 495-501.

5. Podolsky SH, Greene JA, Jones DS. The evolving roles of the medical journal. N Engl J Med 2012: 366: 1457-1461.

6. Sismondo S. Ghosts in the Machine: Publication Planning in the Medical Sciences. Soc Stud Sci 2009: 39(2): 171-198.

7. Ross JS, Hill KP, Egilman DS, Krumholz HM. Guest Authorship and Ghostwriting in Publications Related to Rofecoxib. A Case Study of Industry Documents From Rofecoxib Litigation. JAMA 2008: 299(15): 1800-1812.

8. Liesegang TJ, Schachat AP. Enhanced reporting of potential conflicts of interest: rationale and new form. Am J Ophthalmol 2011: 151: 391-393.

9. Roig F, Borrego A. Políticas de declaración de conflictos de interés en revistas biomédicas españolas de orientación clínica. Rev Esp Doc Cient 2015; 38(3): e091. doi: http://dx.doi.org/10.3989/redc.2015.3.1231.

10. González de Dios J, Flores Canoura A, Jiménez Villa J, Gutiérrez Fuentes JA. Qué revistas médicas españoles leen y cómo se informan los médicos de atención primaria. Aten Primaria 2011: 43(12): 629-637.

11. Banegas JR, Villar F, Graciani A, Rodríguez-Artalejo F. Epidemiología de las enfermedades cardiovasculares en España. Rev Esp Cardiol 2006: Supl 6: 3G-12G.

12. Bhargava N, Qureshi J, Vakil N. Funding source and conflict of interest disclosures by authors and editors in gastroenterology specialty journals. Am J Gastroenterol 2007: 102(6): 1146-1150.

13. Winfurt KP, Seils DM, Tzeng JP, Lin L, Schulman KA, Califf RM. Consistency of financial interest disclosures in the biomedical literature: the case of coronary stents. PloS One 2008: 3: e2128.

14. Klitzman R, Chin LJ, Rifai-Bishjawish H. Disclosures of funding sources and conflicts of interest in published HIV/ AIDS research conducted in developing countries. J Med Ethics 2010: 36: 505-510.

15. Forbes TL. Author disclosure of conflict of interest in vascular surgery journals. J Vasc Surg 2011: 54(3 Suppl): 55S-8S.

16. Hui D, Reddy A, Parsons HA, Bruera E. Reporting of Funding Sources and Conflict of Interest in the Supportive and Palliative Oncology Literature. J Pain Symptom Manage 2012: 44: 421-430.

17. Kang H, Moon JY, Chang Y, Koo Y-M, Koh Y. Current Levels of Conflict of Interest Disclosure in Medical Publications from Korea. J Korean Med Sci 2013; 28(7): 978-982.

18. Bridoux V, Moutel G, Schwarz L, Michot F, Herve C, Tuech JJ. Disclosure of Funding Sources and Conflicts of Interest in Phase III Surgical Trials: Survey of Ten General Surgery Journals. World J Surg 2014: 38(10): 2487-2493.

19. Kopelman AM, Gorelick DA, Appelbaum PS. Disclosures of conflicts of interest in psychiatric review articles. J Nerv Ment Dis 2013: 201(2): 84-87.

20. Riechelmann RP, Wang L, O'Carroll A. Disclosure of conflicts of interest by authors of clinical trials and editorials in oncology. J Clin Oncol 2007: 25: 4642-4647.

21. Lerner TG, Miranda Mda C, Lera AT, Ueda A, Briones B, Del Giglio A, et al. The prevalence and influence of selfreported conflicts of interest by editorial authors of phase III cancer trials. Contemp Clin Trials 2012: 33(5): 1019-1022.

22. Bariani GM, de Celis Ferrari AC, Hoff PM, Krzyzanowska MK, Riechelmann RP. Self-reported conflicts of interest of authors, trial sponsorship, and the interpretation of editorials and related phase III trials in oncology. J Clin Oncol 2013: 31(18): 2289-2295.

23. García Ballarin R, García Asensio M, Martínez Nimatuj I, Galván Lago F. Posicionamientos de sociedades científicas y revisiones de grupos de Trabajo: declaración sobre conflictos de intereses y necesidad de transparencia. Aten Primaria 2014: 46(7): 396.

Recibido: 10 de junio de 2015

Aceptado: 11 de agosto de 2015 